\title{
OFICINA DE ORIENTAÇÃO SEXUAL: RELATO DE EXPERIÊNCIA
}

\author{
Lorena D'Oliveira Gusmão ${ }^{1}$ \\ Flávia Alves Moreira ${ }^{2}$ \\ Leiliane Martins Angelo ${ }^{3}$
}

\section{RESUMO}

A experiência descrita no presente texto refere-se ao projeto de educação em saúde intitulado "Oficina de Orientação Sexual", que desenvolveu um trabalho de orientação para jovens de 12 a 18 anos, no que tange à sexualidade, com o objetivo de promover o desenvolvimento pessoal e social do adolescente, por meio de reflexão, com revisão e ampliação dos conceitos e condutas sobre sexualidade. Dividiram-se as ações do projeto em três etapas: estudo bibliográfico, preparação da comunidade e execução das oficinas. Estabeleceu-se parceria com uma Unidade Básica de Saúde do município de Guanambi, na Bahia. A necessidade desse projeto se deu pela observação do elevado quantitativo de atendimentos a gestantes adolescentes na consulta de pré-natal na unidade de saúde. Justifica-se então, este trabalho, por representar uma experiência de aprendizagem e crescimento pessoal

\footnotetext{
${ }^{1}$ Enfermeira Especialista em Terapia Intensiva pelo Instituto Brasileiro de Pós-Graduação e Extensão (IBPEX). Professora Auxiliar do Departamento de Educação, campus XII da Universidade do Estado da Bahia (UNEB). Coordenadora do Projeto de Extensão Oficina de Orientação Sexual. Brasil. Grupo de pesquisa: Linha de Estudo, Pesquisa e Extensão em Atividade Física. E-mail: lgusmao@uneb.br

${ }^{2}$ Acadêmica do curso de Bacharelado em Enfermagem da UNEB - DEDC XII. Brasil. Monitora Bolsista do Projeto de Extensão Oficina de Orientação Sexual. E-mail: flavia_gbi@hotmail.com.br ${ }^{3}$ Acadêmica do curso de Bacharelado em Enfermagem da UNEB/DEDC-XII. Monitora voluntária do Projeto de Extensão Oficina de Orientação Sexual. E-mail: leili_ane.19@hotmail.com.br
}

\begin{tabular}{l|l|l|l|l}
\hline Revista Extensão \& Cidadania & Vitória da Conquista & v. 1, n. 1 & p. 199-209 & jan./jun. 2013 \\
\hline
\end{tabular}


para os autores e, sobretudo, por proporcionar aos envolvidos aprendizado sobre sexualidade, mediante informações adequadas e atitudes preventivas. Ao considerar a reflexão dos autores sobre sua própria sexualidade, a escuta detalhada das vicissitudes da sexualidade do adolescente e as ponderações sobre os temas abordados durante as atividades das oficinas, a proposta de trabalho resultou em um estreitamento da relação entre educadores e jovens, possibilitando maior cumplicidade e troca de experiências, além da constatação da necessidade de uma política de atenção e acompanhamento ao jovem nas unidades de saúde, com o intuito de ampliar o acesso à informação sobre sexualidade e demais questões que envolvam a saúde do adolescente.

Palavras-chave: Adolescente. Orientação sexual. Sexualidade.

\title{
Sexual orientation workshop: report of experience
}

\begin{abstract}
The experience described in this text refers to the health education project entitled "Workshop on Sexual Orientation", has developed a working guide for youth 12 to 18 years, when it comes to sexuality, which aim to encourage personal development and adolescent's social, through reflection, with revision and extension of concepts and behaviors about sexuality. They split up the actions of the project into three phases: a bibliographic study, preparation and execution of community workshops. It was established partnership with a Basic Health Unit of the municipality of Guanambi, in Bahia. The need for this project came about through the observation of the high quantity of care to pregnant adolescents in the prenatal consultation in the Unit provided. Is then justified this work represents one learning experience of personal growth and learning for authors, and especially for providing involved learning about sexuality trough appropriate information, appropriate and specific preventive actions. As a reflection of the authors about their own sexuality, hearing detailed the vicissitudes of adolescent sexuality and the weights on the topics discussed during the workshop activities, the proposed work has resulted in narrowing of the relationship between educators and young people enabling greater complicity and exchange of experiences, and the finding of a need for policy attention and care to young sensing in healthcare facilities to increase access to information about sexuality and other issues involved in adolescent health.
\end{abstract}

Keywords: Adolescent. Sex orientation. Sexuality. 


\section{Introdução}

No contexto da saúde pública, o bem-estar do adolescente vem incitando a produção de estudos devido à importância social que tem sido dada a essa fase, descrita por Fossa (2003) como um período da vida no qual se destacam a puberdade, transformações e a preparação para o ingresso social na vida adulta. Ao retomar a adolescência, no contexto da saúde pública, o autor destaca-a como um momento de riscos à formação de hábitos, de exposição à violência e substâncias psicoativas e iniciação sexual.

Fossa (2003) determina ainda que esses riscos devam ser enfrentados prioritariamente pelo poder público e por toda a sociedade. Cabe ressaltar que o conhecimento dos adolescentes sobre a vida e a sexualidade não é construído espontaneamente e está vinculado também ao convívio social.

Dessa forma, o processo educativo, como apontam Ruiz et al. (2004), deve ser amplo, envolvendo uma comunicação bilateral, com base no contexto de vida das pessoas e seu propósito deve ser a construção de sujeitos sociais capazes de fazer opções construtivas para suas vidas e para a sociedade.

No contato diário com diversificadas situações vividas pelos adolescentes, durante as atividades acadêmicas de prática supervisionada, nos serviços de saúde, perceberam-se os desafios encontrados por eles nas relações com os parceiros, diante de uma gravidez não planejada ou ainda de uma doença sexualmente transmissível (DST), as dificuldades para assumirem as próprias decisões e, principalmente, os conflitos intensificados pela concepção distorcida da sexualidade, que lhes fora apresentada em algum momento da vida.

Considera-se que os adolescentes têm cada vez mais acesso a informação, porém, muitas vezes, a informação disponível é destituída de um processo de reflexão e desvinculada das orientações apropriadas, o que compromete a tomada de decisões. Assim, o projeto "Oficina de Orientação Sexual" começou a ser delineado com o intuito de abordar 
a Educação Sexual na Unidade Básica de Saúde, rompendo os limites da família e da escola e dividindo com estes a responsabilidade por esta orientação.

Ressalta-se, atualmente, que para a formação adequada do profissional cidadão é imperativa sua efetiva interação com a sociedade, dessa forma a atividade de extensão compreende a prática acadêmica, na qual a universidade responde às demandas da população ao formar o profissional. Sendo assim, nota-se que a sociedade desponta como um espaço privilegiado de produção do conhecimento.

Considera-se que é de suma importância consolidar essa prática como um processo acadêmico definitivo e concretizado em função das exigências da realidade, sendo esta imprescindível para a formação do discente, para a qualificação do docente e para o intercâmbio com a sociedade em questão.

\section{O cenário e sujeitos do projeto}

A Atenção Básica, em conformidade com o descrito na portaria $n^{\circ} 648$, de 28 de março de 2006, busca a promoção da saúde, a prevenção e tratamento de doenças e a redução de danos ou de sofrimentos que possam comprometer a saúde dos indivíduos. Esta é desenvolvida com base no trabalho em equipe, sendo direcionada a populações de territórios bem definidos, no intuito de resolver os problemas de saúde de maior frequência e relevância nestes territórios. Para a realização de tais ações nos municípios deve-se contar com as Unidades Básicas de Saúde.

A Unidade Básica de Saúde na qual se desenvolveu esse projeto está em funcionamento desde 09 de outubro de 1999 e, atualmente, possui uma equipe multiprofissional composta por médico, enfermeiro, cirurgião-dentista, auxiliar de consultório dentário, técnico em enfermagem e agente comunitário de saúde, entre outros funcionários administrativos, atendendo a 904 famílias.

A assistência prestada à população adscrita organiza-se em ações específicas do Ministério da Saúde, abrangendo a saúde do adulto, do 
idoso, da mulher e da criança. Para atendimento dos adolescentes a unidade não dispõe de uma rotina específica destinada a esse público, partindo também da enfermeira da unidade o anseio em formar uma parceria com a Universidade para instituir práticas voltadas aos jovens com o objetivo de aproximá-los da Unidade Básica de Saúde do bairro, integrando-os aos serviços prestados.

\section{O projeto - histórico e formação}

Durante as atividades acadêmicas de prática supervisionada nos serviços de saúde, sob orientação da Enfermeira Docente da Universidade do Estado da Bahia (UNEB), observou-se um elevado quantitativo de atendimentos a gestantes adolescentes no Programa de Humanização no Pré-natal e Nascimento (PHPN), nas unidades de saúde do município, o que denota alto índice de maternidade/ paternidade entre jovens do bairro.

A falta de informações acerca da sexualidade, numa visão ampla, livre de tabus, foi percebida nessa parcela da população, por desconhecerem os métodos contraceptivos ou as Doenças Sexualmente Transmissíveis (DSTs), além do não discernimento dos conceitos de sexualidade e sexo, deixando de refletir a respeito do funcionamento e das necessidades do próprio corpo, ao desconsiderarem seus próprios valores para adotar um perfil de comportamento que é comum ao grupo social a que pertencem.

Dessa forma, pensou-se em estruturar um grupo aberto de jovens com idade entre 12 e 18 anos, para discutir questões acerca da sexualidade, privilegiando informações sobre o corpo humano e suas necessidades básicas, como relacionamento afetivo embasado em troca e compartilhamento e não apenas em doação unilateral, enfrentamento de dilemas como gravidez indesejada ou mesmo contaminação por DSTs e possível discriminação advinda dessas situações, além de conhecimentos sobre os métodos preventivos, os contraceptivos e seu uso adequado. 
Essa ideia foi concretizada sob a forma de um projeto de extensão universitária vinculado ao Núcleo de Pesquisa e Extensão (NUPEX), da Universidade do Estado da Bahia, Campus XII, intitulado "Oficina de Orientação Sexual".

Suplicy (1994) relata que por meio da orientação sexual pode-se prover as lacunas de informação, remover os preconceitos e agitar os conflitos dos adolescentes, abrindo um espaço no qual ele possa falar ou demonstrar suas angústias e medos.

Como estratégia metodológica, utilizaram-se as oficinas e as dinâmicas de grupo, pois se considera que estas possibilitam o envolvimento dos participantes e a troca de experiências, expondo os diferentes olhares e julgamentos acerca de determinadas temáticas. Desta forma, as atividades em campo desenvolveram-se por meio de seis oficinas temáticas, com duração de 2 a 3 horas, nas quais os monitores eram os direcionadores do grupo.

Segundo Carvalho, Rodrigues e Medrado (2005), a oficina é um trabalho estruturado com grupos, sendo enfocado em torno de uma questão central, proposta pelo mesmo, em um dado contexto social, buscando não apenas uma reflexão racional, mas o envolvimento dos sujeitos de modo integral, incluindo formas de pensar, sentir e agir.

Quanto ao processo de elaboração das oficinas, foram objetivos desse projeto: possibilitar a concretização de um espaço favorável ao questionamento dos aspectos específicos da adolescência apresentados pelos jovens e a socialização de experiências; propiciar ao adolescente o reconhecimento do próprio corpo; conscientizálos acerca da importância de uma conduta preventiva vinculada ao início da atividade sexual, por meio da adoção de um comportamento sexual responsável, com o uso adequado dos métodos contraceptivos disponíveis; e problematizar a questão da responsabilidade associada ao relacionamento sexual.

A execução desse projeto ocorreu em três etapas distintas, a saber: etapa de estudo bibliográfico para o preparo do orientador e monitores, por meio de leituras aprofundadas e discussão de todo 
o conteúdo programado para as atividades das oficinas; etapa de preparação da comunidade, na qual houve o envolvimento dos pais, por meio de uma reunião com as famílias para apresentar o projeto e defender a necessidade de promover a orientação sexual aos adolescentes (ainda nessa etapa foram feitas divulgações no bairro por intermédio de cartazes informativos e entrega de convites individuais aos jovens para participarem voluntariamente do projeto); e a última etapa que constituiu o preparo e a execução das oficinas com os temas predeterminados e seguiram uma sequência preestabelecida.

\section{Relato de experiência}

A execução das oficinas com o público-alvo ocorreu em seis momentos distintos, como ditos anteriormente. A oficina 1 abordou o tema "Puberdade e mudanças físicas". Foi preparada uma apresentação em slides com imagens explicativas para discutir com os envolvidos as questões acerca da imagem corporal, mudança comportamental (psicológica) e transição da infância para adolescência. Os adolescentes participaram expondo suas vivências e medos e demonstraram suas expectativas para o futuro.

A oficina 2 teve como tema principal o "Aparelho reprodutor masculino e feminino", porém em virtude dos tabus, as discussões com o grupo tomaram rumos diferenciados, o que não desviou do objetivo principal da atividade de realizar uma abordagem morfofuncional, incluindo higiene corporal e íntima, além de orientações acerca do ciclo menstrual. A troca de conhecimentos entre o grupo foi enriquecedora, pois demonstrou as diferentes experiências vividas por eles no início da puberdade e as dificuldades em lidar com tais mudanças. Peças anatômicas do aparelho reprodutor masculino e feminino foram utilizadas juntamente com apresentação de slides, imagens e vídeos para enriquecer a dinâmica da oficina.

No que concerne aos temas abordados na segunda oficina, considera-se, com base nos estudos de Altmann (2003), que conhecer o corpo da mulheré fundamental para o domínio de seu funcionamento, de 
modo que possa gerenciá-lo no que tange à sua capacidade reprodutiva. Quanto ao corpo do homem, este não necessita de gerenciamento interno, entretanto, ele carece ser conhecido externamente, no intuito de que a técnica apropriada de utilização da camisinha seja congregada, propiciando a prevenção de doenças e da gravidez.

Os temas "Fecundação, Gestação e Parto" foram discutidos na oficina 3 em forma de palestra com utilização de vídeos e imagens, mas, como no grupo não havia gestantes, as discussões giraram em torno de relatos de casos na família e entre os conhecidos.

$O$ encontro seguinte para realização da oficina 4 teve como objeto de explanação o tema "Gravidez indesejada e Aborto", nesta discutiu-se acerca dos problemas de uma gestação precoce, dos métodos contraceptivos disponíveis e das implicações do aborto. Ocorreram demonstrações sobre o uso correto de todos os métodos disponíveis no Sistema Único de Saúde (SUS), com distribuição de preservativo feminino e masculino e orientações quanto à aquisição dos demais métodos.

Ao longo da execução do projeto percebemos que o grupo cresceu e passou a contar com a presença do sexo masculino, contribuindo com o aprimoramento das discussões dos temas abordados, fato que foi nitidamente percebido durante a oficina 5 que tratou do tema "Doenças Sexualmente Transmissíveis". Para execução das atividades foi solicitado ao Centro de Testagem e Aconselhamento de DST/AIDS (CTA), do município de Guanambi, o empréstimo do álbum seriado de DSTs e das próteses dos órgãos genitais feminino e masculino. Estes recursos foram utilizados para reforçar a explanação realizada na oficina anterior e promover a prática do uso correto dos preservativos feminino e masculino, respectivamente.

A última oficina teve como objetivo promover uma discussão acerca da "Violência sexual", definindo seu conceito e debatendo sobre a prática de sexo ou qualquer outro ato contra a vontade de alguém, o que constitui violência, e quando submetido a essa situação o indivíduo tem o amparo legal da justiça. 
Ao término das atividades das oficinas foi realizada uma escuta ativa dos participantes, no que concerne à opinião acerca do trabalho prestado. Os mesmos relataram satisfação com todo o projeto e seu desenvolvimento e demonstraram interesse em manter este elo estabelecido a partir de então, com a disponibilização antecipada em participar de atividades futuras.

Por fim, foi feita também uma reunião com o coordenador e seus monitores para discutir os resultados obtidos e os novos rumos do projeto, no intuito de melhorar o trabalho a ser realizado.

\section{Conclusão}

No transcorrer da construção desse projeto, o método de trabalho escolhido, o cuidado na elaboração das atividades a serem desenvolvidas em cada uma das oficinas, enfim, todo o processo, foi assinalado pelo intuito de despertar o interesse dos adolescentes, de motivar sua participação e, consequentemente, auxiliá-los na absorção do conhecimento repassado.

Considera-se que esse projeto propiciou a construção de um espaço reservado para a problematização de questões relativas à adolescência e à sexualidade trazidas pelos participantes, destacandose o debate acerca da responsabilidade associada ao relacionamento sexual. Nesse sentido, acredita-se na colaboração com a adesão às práticas de comportamento preventivo, em benefício da emancipação dos sujeitos no campo dos direitos sexuais e reprodutivos.

Ao longo do projeto observou-se que os envolvidos demonstraram conhecimento acerca dos temas abordados nas oficinas, o que foi constatado por meio das socializações ocorridas durante o processo. Outro fato que despertou a atenção foi a ampliação considerável do grupo envolvido, inicialmente composto por quatro adolescentes e estendendo-se, ao final, para dez componentes frequentes.

Acrescenta-se que foi possível ainda aproximar o público jovem do contexto de assistência à saúde, prestada pela Unidade Básica, e 
ainda estabelecer uma relação de confiança entre o grupo e a equipe de monitoras, o que favoreceu a troca de conhecimentos. Por fim, considera-se que as oficinas tiveram alta aceitabilidade por parte do público-alvo, com a participação efetiva destes nas atividades propostas.

Ressalta-se, ainda, que esse projeto atendeu às premissas básicas da atividade de extensão, uma vez que respondeu às demandas da população, e propiciou o crescimento técnico-científico de todos os envolvidos, ratificando o espaço social como um espaço privilegiado de produção do conhecimento.

Apesar da satisfação com o trabalho desenvolvido, almejase neste momento ampliar o público favorecido. Considerou-se importante também uma capacitação de toda a equipe da Unidade Básica de Saúde (UBS) envolvida, uma vez que estes são agentes importantes de disseminação de informação em saúde, principalmente no tocante ao Agente Comunitário de Saúde que se configura como um elo entre a UBS e a comunidade assistida.

\section{Referências}

ALTMANN, Helena. Orientação sexual em uma escola: recortes de corpos e de gênero. Cadernos Pagu, Campinas, n. 21, p. 281-315, 2003. Disponível em: < http://www.scielo.br/scielo.php>. Acesso em: 03 jul. 2011.

BRASIL. Ministério da Saúde. Secretaria de Políticas de Saúde. Área de Saúde do Adolescente e do Jovem. Cadernos juventude, saúde e desenvolvimento, Brasilia, v. 1, 1999.

. Departamento de Atenção Básica. Portaria nº. 648, de 28 de março de 2006. Aprova a Política Nacional de Atenção Básica, estabelecendo a revisão de diretrizes e normas para a organização da Atenção Básica para o Programa de Saúde da Família (PSF) e o Programa de Agentes Comunitários de Saúde (PACS). Diário Oficial da União, Poder Executivo, Brasília, 2006. Disponível em: <http:// dtr2001.saude.gov.br/sas/PORTARIAS/Port2006/GM/GM-648. htm>. Acesso em: 23 jun. 2011. 
CARVALHO, Alysson Massote; RODRIGUES, Cristiano Santos; MEDRADO, Kelma Soares. Oficinas em sexualidade humana com adolescentes. Estudos de Psicologia (Natal), Natal, v. 10, n. 3, dez. 2005. Disponível em: <http://www.scielo.br/scielo.php>. Acesso em: 03 jul. 2011.

FOSSA, Ângela Márcia. Educação sexual na escola: um estudo junto a adolescentes. 2003. 189 f. Dissertação (Mestrado em educação) Programa de Pós-Graduação em Educação, Universidade Metodista de Piracicaba, Piracicaba, 2003.

RUIZ, Vanessa Romeiro; LIMA, Alessandra Ribeiro; MACHADO, Ana Lúcia. Educação em saúde para portadores de doença mental: relato de experiência. Revista da Escola de Enfermagem da USP, São Paulo, v. 38, n. 2, jun. 2004. Disponível em: <http://www.scielo.br/scielo. php>. Acesso em: 08 jul. 2011.

SILVA, Macilene; SILVA, Marcelo; ALVES, Maria de Fátima. Sexualidade e adolescência: é preciso vencer os tabus. In: CONGRESSO BRASILEIRO DE EXTENSÃO UNIVERSITÁRIA, 2., 2004, Belo Horizonte. Anais... Belo Horizonte, 2004.

SUPLICY, Marta. Guia de orientação sexual: diretrizes e metodologia. 10. ed. São Paulo: Casa do Psicólogo, 2005. 